\title{
3rd European Conference on Schizophrenia Research: Facts and Visions
}

Dear Colleagues,

Schizophrenia is a very complex disorder and even now, 100 years after it was first described, we know very little about its causes and origins. The available treatments often do not adequately meet the heterogeneous needs of schizophrenia patients. In recent years, controversies about the aetiology of schizophrenia, the significance of the symptomatology, reliable diagnosis and appropriate classification systems and, of course, optimal and personalized treatment have initiated much research in this field and created a vast amount of data. The development of new technologies has provided new insights into some of the pathogenetic pathways of the disorder.

For the above reasons, it is vital to critically discuss recent advances and to regularly update the scientific knowledge about schizophrenia and translate it into clinical practice. The identification of emerging issues for schizophrenia research and, as a consequence, the outlining of a research agenda and initiation of respective collaborations and networking activities are forward-looking tasks for the scientific community. In addition, it is necessary to promote the implementation of evidence-based strategies to meet challenges such as treatment non-adherence, stigma and social exclusion as well as to ensure the quality of care.

The Competence Network on Schizophrenia initiated and has meanwhile established the European Conference on Schizophrenia Research (ECSR) as a forum to promote these comprehensive and significant issues. Accordingly, the scientific programme of this year's 3rd ECSR includes high quality contributions from acknowledged experts, young scientists, and clinicians, all of whom have the common goal of improving the situation of patients. This abstract supplement documents the broad range of topics to be presented in plenary lectures, prearranged and submitted symposia, workshops, and oral presentation and poster sessions. As in previous years, the co-organising psychiatric associations and their respective schizophrenia sections play a valuable role in this joint thematic conference. We are delighted that the Asian Network for Schizophrenia Research has now also become one of our partners.

On behalf of the scientific committee and the organisers, I cordially invite you to participate in this biennial European Schizophrenia Research Conference.

It's a pleasure to welcome you in Berlin!

Sincerely yours,

Wolfgang Gaebel

Congress President and

Speaker of the Competence Network on Schizophrenia

Competence Network on Schizophrenia

c/o Department of Psychiatry and Psychotherapy

Heinrich-Heine-University Düsseldorf

LVR-Klinikum Düsseldorf

Bergische Landstraße 2

40629 Düsseldorf

phone: +49-(0)211-922-2770

info@kompetenznetz-schizophrenie.de

www.kompetenznetz-schizophrenie.de 